\title{
Inequalities in socioeconomic status, behaviors, healthcare services and vulnerability to HIV/STIs between brothel-based and street-based female sex workers in Yunnan, China
}

\section{Guoxi Cai}

Nagasaki University

Yufen Liu

China CDC

Yixiao Lu

Nagasaki University

Jiwen Wu

Fujian Medical University

Jinman Zhuang

Fujian Medical University

Zhijian Hu

Fujian Medical University

Jianping Zhang

Kunming Medical University

Fei He ( $\boldsymbol{\nabla}$ i.fei.he@fjmu.edu.cn )

Fujian Medical University

Research article

Keywords: China, HIV infection, STIs prevalence, epidemiology, sex worker, behaviors, healthcare service

Posted Date: September 21st, 2020

DOl: https://doi.org/10.21203/rs.3.rs-60058/v1

License: @ (i) This work is licensed under a Creative Commons Attribution 4.0 International License.

Read Full License 
Inequalities in socioeconomic status, behaviors, healthcare services and

vulnerability to HIV/STIs between brothel-based and street-based female sex

\section{workers in Yunnan, China}

Guoxi Cai ${ }_{a, b, c}^{\#}$, Yufen Liu ${ }_{d}^{\#}$, Yixiao Lub, Jiwen Wub,e, Jinman Zhuang, Zhijian $\mathrm{Hu}_{\mathrm{f}}$, Jianping Zhang, Fei He$_{\mathrm{f}}{ }_{\mathrm{f}}$

a. Department of International Health, Institute of Tropical Medicine (NEKKEN), Nagasaki University, Nagasaki, 852-8523, Japan

b. Department of Public Health, Nagasaki University Graduate School of Biomedical

Sciences, Nagasaki, 852-8523, Japan

c. Department of Public Health, Nagasaki Prefectural Institute of Environment and Public Health, Nagasaki, 2-1306-11, Japan

d. Director of Policy and Integration Department, National Center for AIDS/STD

Control and Prevention, China CDC, Beijing, 102206, China

e. Department of Human Anatomy and Histoembryology, School of basic medical sciences, Fujian Medical University, Fuzhou, 350108, China

f. Department of Epidemiology and Health Statistics, School of Public Health, Fujian Medical University, Fuzhou, 350108, China; Fujian Provincial Key Laboratory of Environment factors and Cancer; Key Laboratory of Ministry of Education for 
Gastrointestinal Cancer; Fujian Medical University, Fuzhou, 350108, China; Fujian

Digital Institute of Tumor Big Data, Fujian Medical University, Fuzhou 350122,

China

g. Department of the Child-adolescent and Maternal Care of faculty of Public Health,

Kunming Medical University, Yunnan 650500, China

${ }^{\#}$ Guoxi Cai and Yufen Liu contributed equally to this work

* Correspondence:

Corresponding Author: Fei He

E-mail address: i.fei.he@fjmu.edu.cn

Telephone and fax numbers: +8659122862023 


\begin{abstract}
Background: Commercial sex plays a critical role in the transmission of HIV/STI infections in mainland China because female sex workers (FSWs), who tend to be either street-based (SSWs) or brothel-based (BSWs), are extremely prevalent. These two groups had different behaviors and treatment. Few studies investigated due to SSWs group is difficult to reach.
\end{abstract}

Methods: A cross-sectional survey was conducted in Yunnan Province of China with 129 street-based and 185 brothel-based participants. Peer educators conducted anonymous, face-to-face interviews to collect data on socio-demographic characteristics, HIV/STIs-related knowledge, sex work history, sex behaviours, experience of receiving healthcare service, and experience of abuse from clients. Blood samples were taken for HIV and syphilis testing. Urine samples were taken for gonorrhoea and chlamydia testing.

Results: Significant differences on socio-economic characters and HIV/STIs prevalence are found between the two types of FSWs. SSWs are older than BSWs; have less education, more dependents, and more clients in one week; receive less healthcare services; and have a higher prevalence of HIV/STIs. Binary logistic regression model 
results showed that venue for sex trade and experience of HIV testing were significantly associated with the prevalence of HIV/STIs

Conclusions: The SSWs are at lower socio-economic status and have high HIV/STIs mobility, who are more marginalized, receive less healthcare and are, thus, more vulnerable. China's next step in healthcare intervention should focus on the most hard-to-reach-marginalised groups. HIV/STIs testing and socio-psychological support programmes are urgent needed for these neglected people.

Keywords: China, HIV infection, STIs prevalence, epidemiology, sex worker, behaviors, healthcare service

\section{Background}

Even though China has made significant progress in HIV/AIDS control and prevention, the epidemic is a continuing challenge ${ }^{[1]}$. With the changing trends in transmission routes, more than $90 \%$ of new HIV infections was sexually transmitted path, which is now the primary driver ${ }^{[2]}$. Commercial sex, in particular, plays a critical role in the sexual transmission of HIV/STDs in China. Although the epidemic is largely characterised by a low national prevalence of $0.037 \%^{[3]}$, the prevalence of HIV among 
all sex workers was $0.19 \%$ in $201^{[4]}$, with certain regions having higher prevalence, especially in low-fee female sexual workers (FSWs) was much higher at $4.7 \%{ }^{[5]}$.

The sex industry was virtually eliminated in the 1950s in China but re-emerged and be periodic crackdowns since the 1980s. This increasing trend is from the implementation of open-door and market-economic policies, flourishing synchronously with rapid economic development ${ }^{[6,7]}$. Several socioeconomic changes are driving commercial sex in China. Rural-to-urban migration had increased, resulting in more women turning to commercial sex and men engaging in high-risk sex with FSWs. Attitudes towards sex are also becoming more liberal with an increase in premarital and extramarital sex ${ }^{[8,9]}$.It has estimated the number of FSW in China between 2 and 20 million ${ }^{[10-12]}$. Some studies estimated the (FSWs) population to be as high as $3.6 \%$ of the total adult female population (15-49 years old) in some areas of China, higher than the proportions in other Asian countries or anywhere else in the world ${ }^{[13,14]}$

FSWs in China are a heterogeneous group, usually divided by work venue. The two main groups are the brothel-based sex workers (BSWs) who encounter their clients in various entertainment and service establishments (e.g., bars, hotels, guesthouses, saunas, massage parlors, etc.) and the street-based sex workers (SSWs) who solicit on streets or in parks ${ }^{[15]}$. Some studies have estimated the socio-demographics 
characteristics, HIV-related knowledge, risky behaviors, and prevalence of HIV/STIs among BSWs and their clients ${ }^{[16-20]}$. However, less studies ${ }^{[21-25]}$, have looked at the SSWs, because of their high mobility and secrecy resulting in challenges in gaining access to them.

There were few studies ${ }^{[26,27]}$ in China focus on both SSWs and BSWs. Therefore, we have conducted the much deeper comparative study in China that targets both SSWs and BSWs, comparing socioeconomic factors, sexual behaviors and risky practices, like the experience of verbal or physical abuse, HIV/STIs-related knowledge, HIV/STIs prevalence and their influencing factors, and the efficacy of their health-care.

\section{Methods}

\subsection{Study site and field management}

This study was conducted between August and September 2010, in Lingxiang County of Yunnan province, southeast China. Yunnan province has one of the highest HIV prevalence in China, Lingxiang County being one of the epidemic centres ${ }^{[28]}$.

Lingxiang County has a population of 303,600. It borders Myanmar, near

Southeast Asia's Golden Triangle region of high opium production. By the end of 2008, 
1957 HIV/AIDS cases and 143 AIDS-related deaths were reported in Lingxiang. Even though injecting drug users (IDU) are still the predominant transmission route cumulatively $(51.7 \%)$, the proportion of heterosexual transmission among new cases increased rapidly from $13.2 \%$ in 2004 to $59.5 \%$ in 2008 (Lingxiang CDC). Sex work is prevalent in this county, fuelling the concern of HIV transmission from IDUs to the general population. This site was chosen because of the presence of grassroots NGOs working among the FSWs and their active partnership with the local CDC in providing healthcare to the FSWs.

Four FSW peer educators working for an NGO were recruited into the study team. They helped in the mapping out the two FSW groups, recruitment, and interviews using a structured questionnaire after obtaining written informed consent. Blood and urine samples were collected by laboratory staff from the local CDC for HIV/STIs testing. HIV/STIs-related pre-test-post-test counseling, informing of test results, and free STI and Antiretroviral therapy ART treatment are all provided by certified medical staff with the local CDC. This study received approval from the local Yunnan institutional review boards.

\subsection{Mapping and sampling}


All entertainment establishments (brothels) and locations the SSWs solicit were mapped out before the survey. There were 387 brothel-based FSWs in 53 places in four geographical blocks within the county, and $148 \mathrm{SSW}$ roaming the streets in the north-western part of the county.

For the BSWs, two of the four geographical blocks were randomly selected and 185 of $196(94.4 \%)$ FSWs working for all of the 21 massage salons in the blocks were recruited. For the SSWs, time-location sampling and snowball sampling were adopted, and 129 of 148 (87.2\%) participants were recruited. Every participant was provided 20 Yuan (3.5 USD) as an incentive.

\subsection{Data collection instrument}

A structured questionnaire for FSWs based on The Family Health International Behavioral Surveillance Survey (FHI-BSS) 2000(see the supplementary files), was used to collect data on socio-demographic characteristics, HIV/STI-related knowledge, risk perception, sex work history, sex behavior with clients and sex partners, self-report STI symptoms, and experience of receiving healthcare services. Knowledge of transmission and prevention of HIV/AIDS was measured by seven true/false/unknown questions.

One point was given for each correct answer, with the score ranging from 0 to 7 points.

Knowledge about symptoms of other STIs was similarly measured by ten questions, 
with the score ranging from 0 to 10 points. Both focus group discussions and in-depth interviews with FSWs and peer educators were conducted to check the appropriateness of the questionnaire.

\subsection{Laboratory testing}

Blood samples were taken for HIV and syphilis testing. HIV was tested using the enzyme-linked immunosorbent assay (ELISA) for screening and the western blot test for confirmation. Screening for syphilis was performed using the rapid plasma reagin (RPR) test and the treponema pallidum particle agglutination (TPPA) test. Positive RPR and TPPA constitute a diagnosis of active syphilis. Urine samples were taken for gonorrhea and chlamydia testing by real-time fluorescent quantitative PCR (FQ-PCR) assay. Our study outcome was defined as testing positive for one or more of these four

STIs (HIV, syphilis, gonorrhea, and chlamydia).

\subsection{Statistical methods}

Means and proportions compared by using the Mann-Whitney U-test and the

Chi-square test. A binary logistic regression model with crude odds ratios (OR), adjusted OR, and 95\% confidence intervals (CI) of related factors associated with 
HIV/STIs were calculated. All analyses were carried out with the SPSS11.5 (IBM SPSS

Co., USA). $\mathrm{P}<0.05$ was considered statistically significant (two-sided).

\section{Results}

\subsection{Sociodemographic characteristics and sex worker history}

The sociodemographic characteristics of SSWs and BSWs showed in Table 1.

SSWs were older, more of them married, had received less education, and had to support more dependents than BSWs $(\mathrm{P}<0.001)$. They had begun sex work at an older age, had longer sex worker careers $(\mathrm{P}<0.01)$, and received a lower fee per client than BSWs $(\mathrm{P}<0.001)$. Consistently, they solicited many more clients per day $(\mathrm{P}<0.001)$ than their BSW counterparts.

\subsection{Sex behaviors and risky practices}

Risky practices and condom usage described in Table 2. Drug use within the past year and experience of verbal or physical abuse from clients were reported in both groups (SSW versus (vs.) BSW, 6.1 4.5\%, non-significant (NS), and 35.4 vs. 45.5\%, NS) respectively. Condoms were the most used contraceptive method in both groups (87.3 vs. $89.1 \%$, NS), and $8.1 \%$ of SSWs and $19.2 \%$ of BSWs had experienced condom breakage or slippage during the previous week $(\mathrm{P}<0.01)$. The proportion of consistent 
condom use with clients during the previous week was higher among BSWs than among SSWs (98.9 vs. $92.0 \%, \mathrm{P}<0.01)$. Conversely, condom use with a boyfriend or husband was higher in the street-based group (42.1 vs. $31.8 \%, \mathrm{P}<0.05 ; 52.8$ vs. $11.5 \%, \mathrm{P}<0.001$, respectively).

\subsection{HIV/STIs-related knowledge and risk perceptions, healthcare service, HIV}

\section{testing, and self-reported symptoms}

The HIV/AIDS-related knowledge score was high in both FSW groups, although without the significance. Conversely, the score of knowledge on STIs was low, and a significant difference found between the two groups $(\mathrm{P}<0.001)$. Compared with BSWs, SSWs had received less HIV/STIs related health-care services, such as free condom distribution, STIs testing, and treatment, and HIV counseling and testing (37.0 vs. $64.4 \%, \mathrm{P}<0.001$ ) (). About two-thirds of the subjects in both groups had had HIV testing in the past (68.5 vs. $65.5 \%$, NS) (Table 3$)$.

In addition, $32.5 \%$ of SSWs and $37.3 \%$ of BSWs reported having had STIs symptoms during the past year (NS) (abnormal vaginal discharge 21.4 vs. 24.3\%, NS, genital ulcers 2.4 vs. $5.1 \%$, NS, dysuria 15.9 vs. $15.8 \%$, NS, and genital itch 16.8 vs. 29.3, $\mathrm{P}<0.05)$. The risk perception of getting HIV/STIs from clients and of transmitting 
HIV/STIs to clients were both higher among SSWs than among BSWs (93.7 vs. 75.3, $\mathrm{P}<0.001$, and 89.4 vs. $25.1 \%, \mathrm{P}<0.001$, respectively) (Table 3).

\subsection{HIV/STIs prevalence and related risk factors}

Five of the SSWs and none of the BSWs were confirmed to be infected with HIV $(\mathrm{P}<0.01)$. Syphilis prevalence was higher in SSWs $(7.0$ vs. $1.1 \%, \mathrm{P}<0.01)$. Overall, $37.2 \%$ of SSWs and $24.9 \%$ of BSWs were found to be HIV/STIs infected (Table 4 , $\mathrm{P}<0.05)$

A binary logistic regression model developed for all of the participants. As independent variables, the exposure variable of interest and all variables independently associated with the total number of STI infections were included (Table 5). SSWs are at a higher risk of having STIs compare with those BSWs (OR =2.07, 95\% CI 1.22-3.50), HIV testing is a protective factor for HIV/STI infections $(\mathrm{OR}=0.42,95 \% \mathrm{CI} 0.24-0.71)$.

\section{Discussion}

Our results indicated that significant inequalities found between SSWs and BSWs concerning socio-demographics and healthcare service coverage. FSWs both have a high HIV awareness and condom use rate with clients, however, the issue on frequent 
condom slippage or breakage well all existed for BSWs and SSWs. The SSWs received a significantly lower level of healthcare service and they are consistently more vulnerable and suffering from a higher incidence of HIV/STI infections than are BSWs.

Our findings indicate that violence abuse is a significant risk factor for HIV/STIs infections in FSWs population. Consistent with studies in Zimbabwe ${ }^{[28]}$, Swaziland ${ }^{[29]}$, American $^{[30]}$, our results suggest that efforts to reduce violence are a key component of STI/HIV prevention and control in this vulnerable population. Violence Abuse from FSW clients has little studied in China or in other parts of the world. The illegal status of FSWs, the strong stigma and discrimination toward them in Chinese traditional culture, the fear of having their profession unmasked to family and acquaintances, and gender inequality are all factors that have contributed to their marginal situation and their vulnerability to abuse and violence ${ }^{[31]}$. At the same time, human resources are seriously limited within local health sectors in China ${ }^{[32]}$. therefore, most interventions would occur among the BSWs. Compared to BSWs, SSWs usually have no protectors or permanent venue; hence, they are more marginalized ${ }^{[33]}$, usually excluded from public healthcare sectors and surveillance systems and they are much harder to get the protection. 
We also found HIV testing to be a protective factor against HIV/STIs, which suggests that seeking testing should promoted strongly in both groups and that more studies promoting HIV testing should be encouraged in relation to this population ${ }^{[34,35]}$. Promotion of HIV testing and counselling (VCT) through the social media now become a new strategy in mainland China ${ }^{[3,36]}$. In last decades, the number of HIV testing facilities increased from 7,600 to 30,500 and the annual number of HIV tests increased from 45 million to 201 million (from $3.4 \%$ to $14.5 \%$ of the whole population) ) $^{[37]}$.

Our multivariate analysis results highlight the importance of socioeconomic support in relation to HIV/STIs infection. All the three independent factors-work venue, experience of HIV testing, and abuse from clients - are strongly related to the socioeconomic background of FSWs. Most of the previous AIDS-related studies and interventions in China have been limited to biomedical issues only, with ideas for preventive efforts being almost entirely limited to the health sector. Even fewer studies have integrated HIV/STIs-related problems with other public health issues by looking at multiple-sector cooperation or using multi-disciplinary approaches ${ }^{[38,39]}$. Consequently, the generalization of the research findings are usually limited, as well as the efficacy of prevention intervention. Therefore, socio-epidemiologic studies and interventions considering social-economic and sociocultural backgrounds should be encouraged for 
examining future HIV/STIs preventive approaches in China. Social support approaches, such as professional re-training and re-employment support, social support networks, and cooperation with non-health sectors should considered for FSWs.

The difficulty of reaching this population is a challenge for AIDS prevention in many countries. SSWs usually are not included in routine AIDS-related healthcare intervention or sentinel inspection as a result of their high mobility, mistrust of government, and lack of manpower in local healthcare sectors. In current study, our experience in integrating governmental and grassroots resources suggests that involvement of such organizations and peer educators is important, that skill training should be developed for peers, and that the public health sector and NGOs should be encouraged to integrate their work. Many international and national NGOs are dealing with many sensitive healthcare issues, large space remains for promoting cooperation between government sectors and NGOs on HIV/STIs prevention among high-risk groups in China ${ }^{[40,41]}$. Through assigning some of the routine work with FSWs to NGOs, the governmental healthcare can encourage such NGOs to involve themselves through planning, management, and implementation of healthcare services. The NGOs, on the other hand, should feel free to avail themselves of China's CDC laboratories and technical support. 
SSWs were more hard to reach than BSWs, through close cooperation between a local Chinese CDC and grassroots NGO, we gathered both biological and behavior indicators on participants. The participates in this study successfully represented the whole FSWs in Lingxiang County. Based on no one rejected the interview, we thought there was no response bias. However, in the questionnaire, there were some sensitive questions so that the information bias maybe not avoided. In the meantime, we did much more in-depth comparative research from the risky behavior (themselves to the clients and partners) by using the FHIBSS, which use reliable methods to track HIV risk behaviors over time as part of an integrated surveillance system, which monitors various aspects of the epidemic. They are especially useful in providing information on behaviors among sub-populations who may be difficult to reach through traditional household surveys, but who may be at especially high risk for contracting or passing on HIV, especially for sex workers and their clients. Our results, which demonstrate the efficacy of combining resources at various levels to access these hard-to-reach groups, suggested that outreach programs to these kinds of vulnerable groups are of the utmost importance. The main weakness of this study is its limited scale. As described above, this cross-sectional survey conducted only in one spot. Hence, generalization of the results is limited, and more studies on a broader scale needed. 
Though the Chinese health sector has made much progress in health education and interventions with both the general population and high-risk-groups ${ }^{[32,37]}$ efforts to reach the most marginal and highest risk FSWs remain poor. As this is a cross-sectional design study, the next step for researchers is to design an approach for accessing the hard-to-reach FSWs, as interventional studies needed to examine how to improve healthcare toward this vulnerable population.

\section{Conclusion}

The inequality between BSWs and SSWs is significant and quite visible. The Chinese healthcare system has made significant progress on AIDS/STIs-related interventions among high-risk populations, including health education and heightened condom use. However, more particular technical training and instruction on condom usage is need, and evaluation of the efficacy of intervention programs is need. 


\section{Abbreviations}

FSWs:female sex; BSWs:brothel-based sex workers; SSWs:street-based sex workers

\section{Declarations}

\section{Acknowledgments}

The authors wish to thank all those who took part in the study and Non-Governmental Organization:

Bless China International (BCI), who helped us to interview.

\section{Authors' contributions}

G C, Y L and F H designed the study. G C, Y L, and Jp Z collected the data. G C, Y L, Z H and F

$\mathrm{H}$ analyzed the data. G C, Y L, J W, and Jm Z drafted the manuscript. F H contributed to the interpretation of the results and critical revision of the manuscript for important intellectual content and approved the final version of the manuscript. All authors have read and approved the manuscript.

\section{Funding}

This study was supported by grants from the JSPS KAKENHI Grant Number JP21790592. The study was completed by the author independently, and the funding unit did not intervene. 


\section{Availability of data and materials}

Not applicable.

\section{Ethics approval and consent to participate}

Written informed consent was sought from each potential participant. This study was approved by the Biomedical Ethics Review Committee of Yunnan, China.

\section{Consent for publication}

Not Applicable.

\section{Competing interests}

The authors declare that the research was conducted in the absence of any commercial or financial relationships that could be construed as a potential conflict of interest.

\section{References}

1. Ming-Bo H, Li Y, Bing-Yu L, et al. Characterizing the HIV/AIDS Epidemic in the United States and China. International journal of environmental research and public health. 2015;13(1):30.

2. Global information and education on HIV and AIDS (2019).[Available from https://www.avert.org/professionals/hiv-around-world/asia-pacific/china\#footnote12 
qdlewg2].

3. China Health and Family Planning Commission (2015) '2015 China AIDS Response Progress Report' [Available from:

http://www.unaids.org/sites/default/files/country/documents/CHN_narrative_report_2 015.pdf ]

4. UNAIDS data 2019 [Available from:

https://www.unaids.org/en/resources/documents/2019/2019-UNAIDS-data] .

5. Chu Z, Keming R, M DW, et al. High prevalence of HIV and syphilis and associated factors among low-fee female sex workers in mainland China: a cross-sectional study. BMC infectious diseases. 2014;14:255.

6. Tucker J, Ren X, Sapio F. Incarcerated sex workers and HIV prevention in China:

Social suffering and social justice countermeasures. Social Science \& Medicine. 2010; 70(1):121-129.

7. Gil VE, Wang MS, Anderson AF, Lin GM, Wu ZO. Prostitutes, prostitution and STD/HIV transmission in Mainland China. Social Science \& Medicine. 1996;42(1):141-152.

8. Jiantao L, Shiyue L, Hong Y, et al. Early sex initiation and subsequent unsafe sexual behaviors and sex-related risks among female undergraduates in Wuhan, China. 
Asia-Pacific journal of public health. 2015;27(2):21-29.

9. Hehua X, Jiaying X, Zhizi X, et al. Sexual attitudes, sexual behaviors, and use of HIV prevention services among male undergraduate students in Hunan, China: a cross-sectional survey. BMC public health. 2019;19(1):250.

10. Lu W, Ning W, Liyan W, et al. The 2007 Estimates for People at Risk for and Living With HIV in China: Progress and Challenges. Journal of acquired immune deficiency syndromes (1999). 2009;50(4):414-418.

11. Parish WL, Laumann EO, Cohen MS, et al. Population-Based Study of Chlamydial Infection in China: A Hidden Epidemic. Jama. 2003;289(10):1265-1273.

12. Lau M, China's sex industry flourishing despite dangerous conditions and corrupt police. South China Morning Post. [Available from:

https://www.mirror.co.uk/all-about/south-china-morning-post].

13. Vandepitte J, Lyerla R, Dallabetta G, Crabbé F, Alary M, Buvé A. Estimates of the number of female sex workers in different regions of the world. Sexually transmitted infections. 2006;82(3):18-25.

14. Chow EP, Muessig KE, Yuan L, et al. Risk behaviours among female sex workers in China: a systematic review and data synthesis. PloS one. 2015,10(3):e0120595.

15. Shannon K, Strathdee SA, Goldenberg SM, et al. Global epidemiology of HIV 
among female sex workers: influence of structural determinants. Lancet (London, England). 2015;385(9962):55-71.

16. Ma Q, Jiang J, Pan X, et al. Consistent condom use and its correlates among female sex workers at hair salons: a cross-sectional study in Zhejiang province, China. BMC public health. 2017;17(1):910.

17. Guo Y, Xu X, Fu G, et al. Risk behaviours and prevalences of HIV and sexually transmitted infections among female sex workers in various venues in Changzhou, China. International journal of STD \& AIDS. 2017;28(11):1135-1142.

18. Han L, Zhou C, Li Z, et al. Differences in risk behaviours and HIV/STI prevalence between low-fee and medium-fee female sex workers in three provinces in China. Sexually transmitted infections. 2016;92(4):309-315.

19. Su S, Chow EP, Muessig KE, et al. Sustained high prevalence of viral hepatitis and sexually transmissible infections among female sex workers in China: a systematic review and meta-analysis. BMC infectious diseases. 2016;16:2.

20. Zeng H, Zhang L, Zhao Y, et al. HIV prevention among street-based sex workers (SSWs) in Chongqing, China: interviews with SSWs, clients and healthcare providers. Health \& social care in the community. 2016;24(6):e173-e80.

21. Zeng H, Zhao Y, Meng S, et al. Exploring HIV prevention strategies among 
street-based female sex workers in Chongqing, China. International journal of environmental research and public health.2015; 12(1):855-70.

22. Chang RC, Hail-Jares K, Zheng H, He N, Bouey JZH. Mitigating circumstances: A model-based analysis of associations between risk environment and infrequent condom use among Chinese street-based sex workers. PloS one.2018;13(5):e0195982.

23. Okafor UO, Crutzen R, Ifeanyi O, Adebajo S, Van den Borne H. HIV prevalence and high-risk behaviour of young brothel and non-brothel based female sex workers in Nigeria. BMC research notes. 2017;10(1):380.

24. Basnyat I. Lived experiences of street-based female sex workers in Kathmandu: implications for health intervention strategies. Culture, health \& sexuality. 2014; 16(9):1040-1051.

25. Mastrocola EL, Taylor AK, Chew-Graham C. Access to healthcare for long-term conditions in women involved in street-based prostitution: a qualitative study. BMC family practice. $2015 ; 16: 118$.

26. Li Y, Detels R, Lin P, et al. Difference in risk behaviors and STD prevalence between street-based and establishment-based FSWs in Guangdong Province, China. AIDS and behavior. 2012;16(4):943-951. 
27. Li J, Chen XS, Merli MG, Weir SS, Henderson GE. Systematic differences in risk behaviors and syphilis prevalence across types of female sex workers: a preliminary study in Liuzhou, China. Sexually transmitted diseases. 2012;39(3):195-200.

28. Qiao S, Yuk-Ha, Tsang E, Wilkinson JS, Lipeleke F, Li X. "In Zimbabwe there is nothing for us": sex work and vulnerability of HIV infection among male sex workers in Zimbabwe. AIDS care. 2019;31(9):1124-1130.

29. Berger BO, Grosso A, Adams D, et al. The Prevalence and Correlates of Physical and Sexual Violence Affecting Female Sex Workers in Swaziland. Journal of interpersonal violence. 2018;33(17):2745-2766.

30. DiStefano Anthony S, Gill Jasmeet K, Hubach Randolph D, Cayetano Reggie T, Hilbert Cary J. HIV testing in an ethnically diverse sample of American university students: associations with violence/abuse and covariates. Journal of behavioral medicine. 2014;37(5):1030-1046.

31. Haile, Sofia. "HIV/AIDS in Yunnan Province: A Study of Stigma and Support" (2013).Independent Study Project (ISP) Collection. [Available from:https://digitalcollections.sit.edu/isp_collection/1724].

32. Wu Z. HIV/AIDS in China:Beyond the Numbers. (2016).

33. Hong Y, Zhang C, Li X, Zhou Y, Guo W. Female sex workers and their gatekeepers 
in China: implications for HIV/STI prevention. Qualitative health research. 2014; 24(10):1431-1439.

34. Ulrich M, Jasmin O, Marc G, Kai E, Karin W, Andreas W. Risk factors for HIV and STI diagnosis in a community-based HIV/STI testing and counselling site for men having sex with men (MSM) in a large German city in 2011-2012. BMC infectious diseases. 2015;15:14.

35. Wilson Chialepeh N, Sathiyasusuman A. Associated Risk Factors of STIs and Multiple Sexual Relationships among Youths in Malawi. PloS one. 2015; 10(8):e0134286.

36. Liu Y, Sun X, Qian HZ, et al. Qualitative Assessment of Barriers and Facilitators of Access to HIV Testing Among Men Who Have Sex with Men in China. AIDS patient care and STDs. 2015;29(9):481-489.

37. UNAIDS Country Progress Report. China. Global AIDS monitoring 2018 [Available from:https://www.aidsdatahub.org/global-aids-monitoring-2018-unaids-2017].

38. Haregu TN, Setswe G, Elliott J, Oldenburg B. National Responses to HIV/AIDS and Non-Communicable Diseases in Developing Countries: Analysis of Strategic Parallels and Differences. Journal of public health research. 2014;3(1):99. 
39. Muessig KE, Smith MK, Maman S, Huang Y, Chen XS. Advancing the prevention agenda for HIV and other sexually transmitted infections in south China: social science research to inform effective public health interventions. AIDS and behavior . $2014 ; 18(2): 222-231$.

40. Wang D, Mei G, Xu X, et al. Chinese non-governmental organizations involved in HIV/AIDS prevention and control: Intra-organizational social capital as a new analytical perspective. Bioscience trends. 2016;10(5):418-423.

41. Lau JTF, Wang Z, Kim Y, et al. Low sustainability, poor governance, and other challenges encountered by grassroots non-governmental organizations targeting HIV prevention for men who have sex with men in China - a nation-wide study. AIDS Care. 2017;29(12):1480-1490.

\begin{tabular}{|c|c|c|c|c|c|}
\hline & \multicolumn{2}{|c|}{ Street-based (n1) } & \multicolumn{2}{|c|}{ Brothel-based (n2) } & \multirow[t]{3}{*}{$\mathrm{P}$ value } \\
\hline & & (mean, SD)/ & & (mean, SD)/ & \\
\hline Variable & & (median, IQR)/\% & & (median, IQR)/\% & \\
\hline Age (mean, $\mathrm{SD},{ }^{* *}$ years) & 129 & $34.0(8.3)$ & 182 & $25.1(6.0)$ & $<0.001^{\mathrm{a}}$ \\
\hline Age of sex debut (mean, SD, year) & 127 & $20.0(2.8)$ & 178 & $18.1(2.4)$ & $<0.001^{\mathrm{a}}$ \\
\hline
\end{tabular}


Age of starting sex work (mean, SD, year)

Length of time as an FSW (median, IQR, ${ }^{* * *}$

year)

Charge per client (mean, $\mathrm{SD}, \mathrm{RMB}^{\mathrm{c}}$ )

$25.0(13.0)$

177

$102.0(34.0)$

$<0.001^{\mathrm{a}}$

No. of clients in last working day (median, IQR,

person)

No. of clients in last working day (mean, SD)

Ethnic group
Divorced

Widowed

Educational level 
No. of dependents

1 or 2

3 or over
51

69
7.0

39.5

53.5
57

80

45
31.3

44.0

24.7

${ }^{a}$ Chi-square test (2-sided)

${ }^{\mathrm{b}}$ Mann-Whitney U test (2-tailed)

c1 USD=6.5 RMB

Note: Percentages do not always add up to $100 \%$ because of rounding down; missing data is not calculated.

${ }^{*}$ FSWs, female sex workers; ${ }^{* *} \mathrm{SD}$, standard deviation; ${ }^{* * *} \mathrm{IQR}$, interquartile range.

Table 2. HIV/STIs-related risks and history of sex work in street-based and brothel-based FSWs in Yunnan, China, 2010.

\begin{tabular}{|c|c|c|c|c|}
\hline \multirow{5}{*}{ Items } & \multirow{3}{*}{ (n1) } & Brothel based & \multirow[t]{3}{*}{ Street/Brothel } & \multirow{5}{*}{$\mathrm{P}_{\text {value }}{ }^{1}$} \\
\hline & & (n2) & & \\
\hline & & & & \\
\hline & Frequency/ & Frequency/ & Crude OR & \\
\hline & Percentage $(\%)$ & Percentage (\%) & $(95 \% \mathrm{CI})$ & \\
\hline
\end{tabular}

Drug use in past 1 year $(\mathrm{n} 1=33, \mathrm{n} 2=177)$ 
No

Yes

$45(35.4)$

Frequency of condom use with clients in past 1 week $(n 1=125, n 2=177)$

Every time

$115(92.0)$

$10(8)$

Frequency of condom use with boyfriend(s) in 1 week $(n 1=57, n 2=107)$

Every time

24(42.1)

$33(57.9)$

Sometimes

Frequency of condom use with husband in past 1 week $(n 1=53, n 2=26)$

Every time

Sometimes
$28(52.8)$

25(47.2)
$97(54.5)$

$1.0(\mathrm{ref})$

$81(45.5) \quad 0.66(0.41-1.05)$ 
${ }^{1}$ Chi-square test (2-sided)

Table 3. HIV/STIs healthcare service, knowledge, and risk perception of street-based and brothel-based FSWs* in Yunnan, China, 2010

\begin{tabular}{lll}
\hline Street-based (n1=129) & Brothel-based (n2=185) & \\
\cline { 2 - 3 } & & \\
Frequency/Percentage(\%) & Frequency/Percentage(\%) & P value $^{1}$ \\
& & \\
\hline
\end{tabular}

Ever receive any AIDS-related health service (n1=127,

n2=177) 


\begin{tabular}{|c|c|c|c|}
\hline No & $80(63.0)$ & $63(35.6)$ & 1.0 \\
\hline Yes & $47(37.0)$ & $114(64.4)$ & $0.33(0.20-0.52)$ \\
\hline
\end{tabular}

Experience of HIV testing $(\mathrm{n} 1=127, \mathrm{n} 2=177)$

\begin{tabular}{|c|c|c|c|}
\hline No & $40(31.5)$ & $61(34.5)$ & 1 \\
\hline Yes & $87(68.5)$ & $116(65.5)$ & $1.14(0.70-1.86)$ \\
\hline
\end{tabular}

Self-reported STIs symptoms in past 1 year $(\mathrm{n} 1=126$,

$\mathrm{n} 2=177$ )

No

$85(67.5)$

111(62.7)

1
Yes
41(32.5)
$66(37.3)$
$0.81(0.50-1.31)$

Know some PLWHA or people who died of AIDS

$<0.001^{1}$

$(\mathrm{n} 1=112, \mathrm{n} 2=177)$

No

53(47.3)

165(93.2)

1

Yes

59(52.7)

12(6.8)

$15.31(7.65-30.63)$

Correctly answered all 7 HIV-related items (\%) $(n 1=126$, n2=177)

$0.800^{1}$

AIDS knowledge score

$$
38(30.2)
$$

1

less than 7

AIDS knowledge score is $7 \quad 88(69.8)$

$126(71.2)$

$0.94(0.57-1.55)$

Knowledge score of STIs symptoms $0-2=0,3-10=1(n 1=120, n 2=170)$

$<0.001^{1}$ 


\begin{tabular}{|c|c|c|c|}
\hline $0-2$ & $85(70.8)$ & $62(36.5)$ & 1 \\
\hline 10-mar & $35(29.2)$ & $108(63.5)$ & $0.24(0.14-0.39)$ \\
\hline
\end{tabular}

Perception: risk of getting HIV/STIs from clients $(\mathbf{n} 1=126, \mathbf{n} 2=170)$

No $\quad 8(6.3) \quad 42(24.7)$

$\begin{array}{lll}\text { Yes } & 118(93.7) & 128(75.3)\end{array}$

Perception: risk of transmitting HIV/STIs to client $(n 1=123, \mathrm{n} 2=171)$

$<0.001^{1}$

$\begin{array}{llll}\text { No } & 13(10.6) & 128(74.9) & 1\end{array}$

$\begin{array}{lll}\text { Yes } & 110(89.4) & 43(25.1)\end{array}$

${ }^{1}$ Chi-square test (2-sided), b: Mann-Whitney U test

(2-tailed)

*FSWs: female sex

workers

Table 4. HIV/STIs test results of street-based and brothel-based FSWs* in Yunnan, China, 2010.

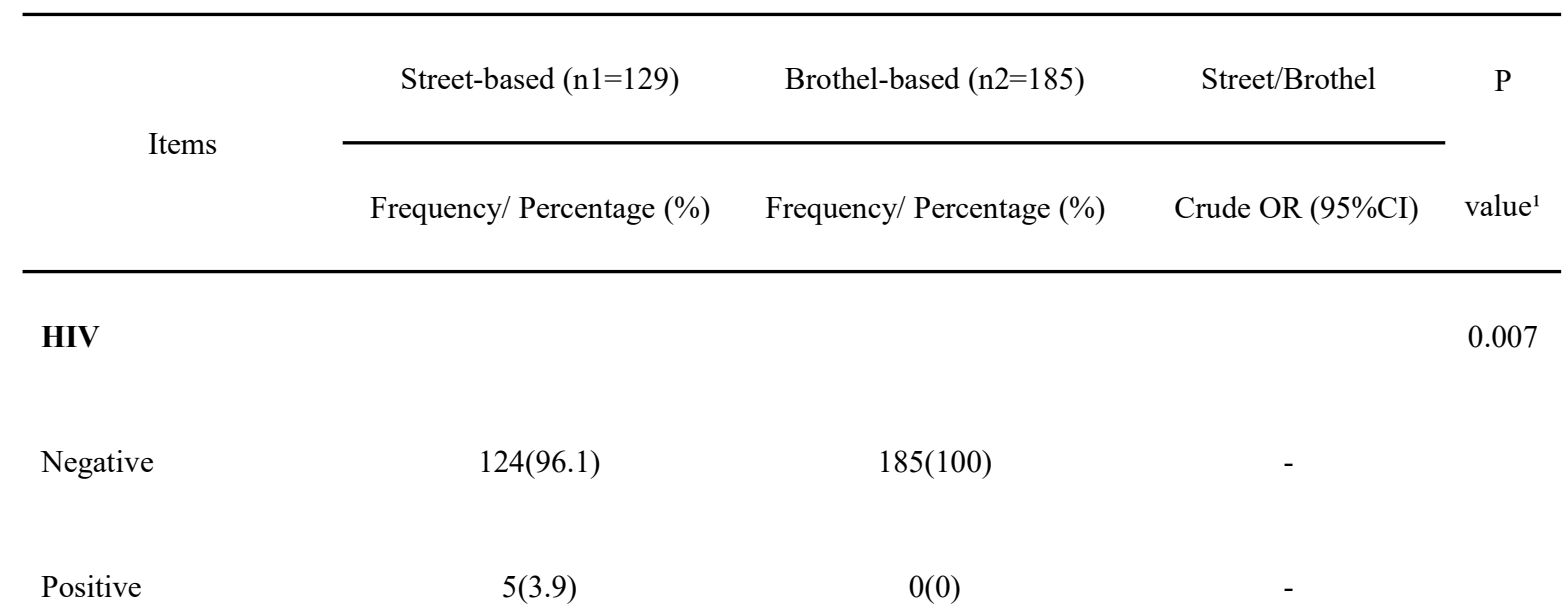


Negative

Positive

Gonorrhoea

Negative

Positive

Chlamydia

Negative

Positive

$30(23.3)$

Any of the upper 4 STIs tested positive

No

$81(62.8)$

$48(37.2)$
183(98.9)

2(1.1)

$6.86(1.46-32.31)$

0.248

167(90.3)

18(9.7)

$1.51(0.75-3.02)$

$149(80.5)$

1

36(19.5)

$1.25(0.73-2.17)$

0.019

139(75.1)

46(24.9)

$1.79(1.10-2.92)$

${ }^{1}$ Chi-square test (2-sided)

*FSWs: female sex workers 
Table 5. Factors associated with STIs prevalence of street-based and brothel-based FSWs* in Yunnan, China, 2010.

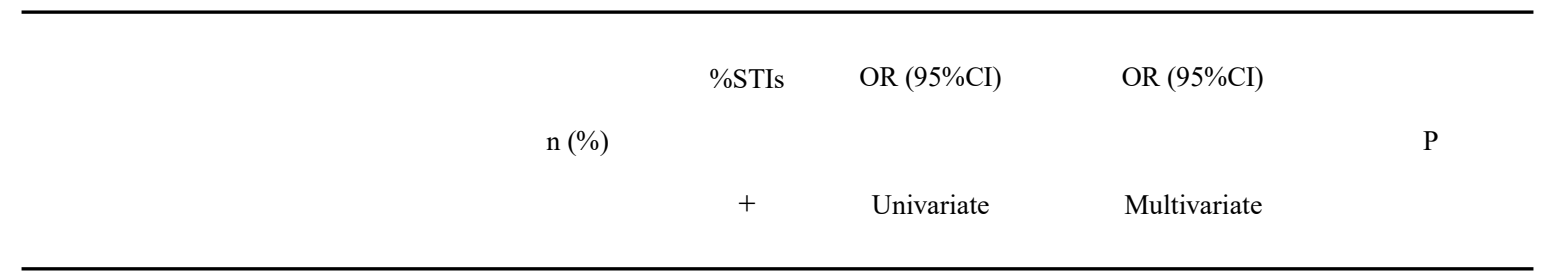

Street01(n=314)

Brothel-based

Street-based
$129(41.1)$

24.9

185 (58.9)
37.2

$1.79(1.10-2.92)$

$2.07(1.22-3.50)$ 
Dependence $(\mathrm{n}=\mathbf{3 1 1})$

$\begin{array}{lccc}0 & 66(21.0) & 24.2 & 1.0 \\ 1 \text { or } 2 & 131(41.7) & 31.3 & 1.42(0.73-2.79) \\ 3 \text { or over } & 114(36.3) & 31.6 & 1.44(0.73-2.87)\end{array}$

Ever receive any AIDS-related health service $(n=304)$

$143(53.0) \quad 25.2 \quad 1$

No

$161(47.0) \quad 34.8$

$1.59(0.96-2.61)$

\section{Experience of HIV testing}

(n=304)

No

101(33.2) $\quad 23.6$

1

1

0.001

Yes

$203(66.8)$

44.6

$0.80(0.23-0.64)$

$0.42(0.24-0.71)$

Charge per client (in RMB) $(n=305)$

$0-50$

$131(43.0)$

36.6

$1.76(1.07-2.89)$

$>50$

$174(57.0)$

24.7

1.0

Verbal or physical abuse by clients in past 1 month $(n=305)$

No

26.3

1

1

0.067

Yes

$126(41.3)$

35.7

$1.56(0.95-2.56)$

$1.64(0.97-2.79)$

Frequency of condom use with clients in 1 week $(n=302)$ 
Every time

Sometime

$12(4.0)$

58.3

$3.43(1.06-11.12)$

\section{Correctly answer to all of the 7 HIV-related items (\%)}

$(\mathbf{n}=\mathbf{3 0 3})$

AIDS knowledge score is less than

$89(29.4) \quad 38.2 \quad 1$

AIDS knowledge score is 7

$214(70.6)$

27.6

$062(0.37-1.04)$

Note: The multivariate model includes the exposure variables of interest, and variables independently associated with STI

infections.

${ }^{*}$ Female sex workers 


\section{Supplementary Files}

This is a list of supplementary files associated with this preprint. Click to download.

- Questionnaire.pdf 